\title{
Relevant muscle selection for needle electromyography examination in polio survivors
}

\author{
Halit Fidancı ${ }^{1,2} \odot$, İlker Öztürk²๑, Zülfikar Arlıer® \\ ${ }^{1}$ Department of Neurology, Division of Clinical Neurophysiology, University of Health Sciences, Adana City Training and Research \\ Hospital, Adana, Turkey \\ ${ }^{2}$ Department of Neurology, University of Health Sciences, Adana City Training and Research Hospital, Adana, Turkey
}

\begin{abstract}
Objectives: Needle electromyography (EMG) has an important role in the diagnosis of poliomyelitis. Since needle EMG is a painful and time consuming procedure, selecting the most effective muscles for needle EMG is important. We aimed to determine the clinically or subclinically affected regions in polio survivors by examining the minimum number of muscles with EMG.

Methods: Polio survivors with weakness in at least one limb were included in this retrospective cohort study. The extremities were divided into two groups: 1) Group 1: The extremities with Medical Research Council score of $\leq 1$ in at least one muscle, 2) Group 2: Clinically unaffected or mildly to moderately affected extremities that do not meet the criteria of Group 1. The needle EMG findings of the muscles were analyzed. Results: Twenty-nine polio survivors were included in the study. Needle EMG findings of 352 muscles were reviewed. Needle EMG findings in 57 lower extremities and thirty-two upper extremities were analyzed. There was no upper extremity meeting the criteria of Group 1. Thirty-eight lower extremities were included in Group 1. The amplitudes and durations of motor unit action potentials (MUAPs) were not different between the muscles of the Group 1 lower extremities $(p>0.05)$. Among the muscles of Group 2 upper and lower extremities, the amplitude and duration of MUAPs were higher in the deltoid and the vastus lateralis muscles compared to the other muscles, respectively ( $p=0.002$ and $p=0.003$ for upper extremity muscles; $p=0.005$ and $p<0.001$ for lower extremity muscles).

Conclusions: Using the needle EMG findings, an algorithm was made to determine the affected regions. Thus, the affected regions can be identified by applying needle EMG to a minimum number of muscles.

Keywords: electrodiagnosis, needle electromyography, poliomyelitis, polio survivors
\end{abstract}

$P^{\prime}$ oliomyelitis is a disease caused by RNA enterovirus affecting the brain and the motor neurons of the spinal cord and brainstem. Poliovirus caused many deaths and disabilities in the 1940s and 1950 s; fortunately, it is rarely seen today with the development of vaccines $[1,2]$. On the other hand, patients with polio sequelae continue to have health problems such as musculoskeletal problems or late neuromuscular deterioration. Poliovirus affects most motor neurons and destroys about $50 \%$ of these motor neurons $[1,3,4]$. After this acute phase, the disease may remain stable over the years. In some patients, late neuromuscular deterioration or post-polio syndrome may develop in addition to the polio sequelae 
after years.

The cause of the neuromuscular deterioration is unknown, but it is thought that factors such as excessive exercise, chronic poliovirus infection, changes in muscle fiber metabolism, or immune-mediated mechanisms may be triggers $[1,5,6]$. Regardless of the cause, motor neurons are damaged faster than normal aging $[7,8]$.

It may be important to demonstrate the clinical and subclinical involvement with needle electromyography (EMG) [9]. Thus, protection of motor neurons or axons can be provided by physical therapy strategies. Needle EMG is a painful and time-consuming electrodiagnostic test, so it is not possible to evaluate all the muscles of a polio survivor with needle EMG. Therefore, it was aimed to select the most effective muscles for needle EMG examination and find the clinically / subclinically affected extremities in polio survivors by examining the minimum number of muscles with needle EMG.

\section{METHODS}

\section{Subjects}

Patients with polio sequelae admitted to EMG laboratory between July 2018 and December 2019 were included in this retrospective cohort study. Ethics committee approval was received from the ethics committee of Adana City Training and Research Hospital (number: 46/636). The clinical features and electrodiagnostic findings of patients with a history of poliomyelitis and weakness in at least one limb were reviewed. The patients who had polyneuropathy or a disease that could cause polyneuropathies such as diabetes mellitus and those with a history of surgery due to lumbosacral and cervical radiculopathy or findings suggesting significant cervical or lumbosacral radiculopathy in magnetic resonance imaging or computer tomography were excluded from the study. Medical research council (MRC) scale was used for muscle strength assessment $[10,11]$. The MRC scores of arm abduction, elbow flexion, elbow extension, wrist extension, and finger abduction were analyzed in the upper extremities. MRC scores of hip flexion, knee flexion, knee extension, ankle flexion, and ankle dorsiflexion were analyzed in the lower extremities. The muscles with an MRC score of 0 and 1 cannot resist a force $[10,11]$. For this reason, it is difficult to obtain the motor unit action potential (MUAP) with needle EMG in muscles with MRC scores $\leq 1$. Therefore, the extremities were divided into two groups: 1) Group 1 was defined as clinically severely affected extremities with an MRC score of $\leq 1$ in at least one muscle examined, 2) Clinically unaffected or mildly to moderately affected extremities that did not meet the criteria of Group 1 were defined as Group 2.

\section{Electrodiagnostic Tests}

The nerve conduction studies and the needle EMG were performed with the Cadwell Sierra Summit EMG unit (Cadwell Laboratories, Kennewick, Washington, USA). Electrodiagnostic tests were performed when the extremity temperature was $\geq 32^{\circ} \mathrm{C}$; otherwise, the extremities were heated. Surface electrodes were used for stimulation and recording. Median, ulnar, posterior tibial, peroneal, sural sensory, and motor nerve conduction studies were performed using conventional methods. Band-pass filters for sensory and motor nerve conduction studies were set at $20 \mathrm{~Hz}$ to $2 \mathrm{kHz}$ and $20 \mathrm{~Hz}$ to $10 \mathrm{kHz}$, respectively. For the reference values of nerve conduction studies, the reference values recommended by the American Association of Neuromuscular \& Electrodiagnostic Medicine were considered [12]. Needle EMG was performed with a concentric needle electrode (length $=50 \mathrm{~mm}$, diameter $=0.46 \mathrm{~mm}$, Bionen Medical Devices, Florence, Italy). Low and high-frequency filters for needle EMG were $10 \mathrm{~Hz}$ and $10 \mathrm{kHz}$, respectively. For the analysis of spontaneous activity, sensitivity and sweep speed were set to $100 \mathrm{uV} /$ division and $10 \mathrm{~ms} /$ division, respectively. The low and high filters for the analysis of the MUAPs were 200-1000 uV/division and $10 \mathrm{~ms} /$ division, respectively. In polio survivors with lower limb weakness, needle EMG was applied to the muscles of both lower extremities and the muscles of one upper extremity. In addition, if upper extremity weakness was present, needle EMG was applied to the muscles of both upper extremities. Although the trapezius muscle is innervated by both the 11th cranial nerve and the cervical segments, needle EMG was applied to the trapezius muscle to determine cranial nerve involvement. Needle EMG was performed visually. Positive sharp waves (PSWs) and fibrillation potentials (FP) 
and fasciculation potentials were carefully examined. The severity of PSWs and FPs were evaluated as follows [13]: (0) Absence of PSWs and FPs, (1) Single PSW or FP in at least two areas, (2) A moderate number of PSWs or FPs in three or four areas, (3) PSWs or FPs in all areas and (4) PSWs or FPs filling the screen in all areas. During mild muscle contraction, at least 10 MUAPs were analyzed. If the amplitude of the MUAPs were $>4 \mathrm{mV}$ or the duration of the MUAPs was $>15 \mathrm{~ms}$, the MUAPs were considered neurogenic. At least 10 MUAPs were attempted to be analyzed from one muscle, but if the MUAPs were few, these MUAPs were taken into account. Among the MUAPs obtained from a muscle, those with the highest amplitude and longest duration were analyzed. In the case of neurogenic needle EMG findings in two muscles innervated by different segments and different nerves, this region was considered to be affected.

\section{Statistical Analysis}

The Shapiro-Wilk test was used to determine the distribution of the data. Comparisons were made using the Friedman test and Wilcoxon signed-rank test for dependent samples. Bonferroni correction was used as post hoc analysis. Pearson's Chi-squared test was used to analyze categorical variables. Mean \pm standard deviation (SD) and median of numeric data were calculated for descriptive statistics. Statistical Package for the Social Sciences (SPSS IBM Corp; Armonk, NY, USA) 22.0 was used to perform the statistical analysis.

\section{RESULTS}

Thirty-two patients with polio sequelae were evaluated. Two patients with diabetes mellitus and one with a history of surgery due to lumbosacral radiculopathy were excluded, so twenty-nine polio survivors were included in the study. The clinical findings of seven patients were compatible with post-polio syndrome. Clinical findings and nerve conduction studies of two patients were consistent with carpal tunnel syndrome, and the needle EMG findings of the abductor pollicis brevis muscles of these patients were excluded. Nineteen patients $(66 \%)$ were male. The mean age of the patients was $45.6 \pm 7.5$ (range 31-59) years. The mean age of the patients with acute poliomyelitis was $2.0 \pm 1.2$ (range 1-5) years. There was no patient with nerve conduction finding consistent with polyneuropathy. Fourteen patients had weakness in only one lower extremity, while 12 patients had weakness in both lower extremities. Ten of 12 patients with bilateral lower limb weakness had an asymmetric weakness. There were two patients with weakness in one upper and bilateral lower extremities and one patient with weakness in the left upper and right lower extremities. The compound muscle action potential (CMAP) of 56 posterior tibial nerves and 54 peroneal nerves were evaluated. The CMAP amplitude was reduced in $13(23 \%)$ of 56 posterior tibial nerves and not obtained in $14(26 \%)$ of 54 peroneal nerves. Seventeen (59\%) of 29 patients had reduced CMAP amplitude or

Table 1. Needle EMG findings in the muscles of the Group 1 extremities

\begin{tabular}{lccc}
\hline Muscles & $\begin{array}{c}\text { Number of muscles with } \\
\text { abnormal needle EMG } \\
\text { findings*// total number of } \\
\text { muscles examined in } \\
\text { needle EMG (\%) }\end{array}$ & $\begin{array}{c}\text { Mean MUAP amplitude } \\
\mathbf{m V} \text { (median) (number) }\end{array}$ & $\begin{array}{c}\text { Mean MUAP duration ms } \\
\text { (median) (number) }\end{array}$ \\
\hline TA & $32 / 35(91 \%)$ & $7.7 \pm 3.1(8.9)(\mathrm{n}=22)$ & $20.3 \pm 6.7(20)(\mathrm{n}=22)$ \\
MG & $36 / 37(97 \%)$ & $7.7 \pm 2.6(8)(\mathrm{n}=16)$ & $19.9 \pm 6.1(19)(\mathrm{n}=16)$ \\
PL & $6 / 6(100 \%)$ & $5.7 \pm 1.5(6)(\mathrm{n}=3)$ & $18.0 \pm 1.7(17)(\mathrm{n}=3)$ \\
VL & $31 / 32(97 \%)$ & $9.2 \pm 3.5(8.5)(\mathrm{n}=16)$ & $21.6 \pm 4.7(20)(\mathrm{n}=16)$ \\
IP & $30 / 32(94 \%)$ & $7.6 \pm 2.7(8)(\mathrm{n}=21)$ & $21.1 \pm 3.9(20)(\mathrm{n}=21)$ \\
& & $\boldsymbol{p}=\mathbf{0 . 4 0 6}$ & $\boldsymbol{p}=\mathbf{0 . 1 9 1}$ \\
\hline
\end{tabular}

EMG $=$ electromyography, $\mathrm{MRC}=$ Medical Research Council, MUAP = motor unit action potential, TA = tibialis anterior, $\mathrm{MG}=$ medial gastrocnemius, $\mathrm{PL}=$ peroneus longus, $\mathrm{VL}=$ vastus lateralis, $\mathrm{IP}=$ iliopsoas. $*$ Needle EMG findings were considered abnormal if neurogenic MUAP was detected or if MUAP was not obtained during voluntary mild muscle contraction. Friedman test and Wilcoxon signed-rank test were used to compare MUAP amplitudes and duration of muscles. Boferroni correction was used for post hoc analysis. P values $<0.05$ were considered statistically significant. 


\section{Table 2. Needle EMG findings in the muscles of the Group 2 extremities}

\begin{tabular}{lccc}
\hline Muscles & $\begin{array}{c}\text { Number of muscles } \\
\text { with neurogenic needle } \\
\text { EMG findings / total } \\
\text { number of muscles } \\
\text { examined in needle } \\
\text { EMG (\%) }\end{array}$ & $\begin{array}{c}\text { Mean MUAP amplitude } \\
\text { mV (median) }\end{array}$ & $\begin{array}{c}\text { Mean MUAP duration } \\
\text { ms (median) }\end{array}$ \\
\hline TA $(\mathrm{n}=18)$ & $12 / 18(67 \%)$ & $5.2 \pm 3.5(4.8)$ & $18.5 \pm 6.7(18.5)$ \\
\hline MG $(\mathrm{n}=19)$ & $9 / 19(47 \%)$ & $3.7 \pm 3.1(1.5)$ & $15.7 \pm 6.9(13)$ \\
\hline PL $(\mathrm{n}=6)$ & $2 / 6(33 \%)$ & $2.9 \pm 2.9(1.3)$ & $14.8 \pm 4.2(13.5)$ \\
VL $(\mathrm{n}=18)$ & $17 / 18(94 \%)$ & $8.9 \pm 3.2(8.3)$ & $27.8 \pm 8.4(28)$ \\
\hline IP $(\mathrm{n}=13)$ & $10 / 13(77 \%)$ & $6.4 \pm 3.2(7)$ & $21.5 \pm 6.4(20)$ \\
\hline FDI $(\mathrm{n}=32)$ & $12 / 32(38 \%)$ & $\boldsymbol{p}=\mathbf{0 . 0 0 2}$ & $\boldsymbol{p}=\mathbf{0 . 0 0 3}$ \\
\hline APB $(\mathrm{n}=8)$ & $2 / 8(25 \%)$ & $2.9 \pm 2.7(1)$ & $13.8 \pm 4.3(12.5)$ \\
Triceps brachii $(\mathrm{n}=18)$ & $8 / 18(44 \%)$ & $3.9 \pm 3.2(2.8)$ & $12.3 \pm 1.9(12)$ \\
\hline Biceps brachii $(\mathrm{n}=31)$ & $8 / 31(26 \%)$ & $2.6 \pm 2.6(1.5)$ & $14.3 \pm 4.1(13.5)$ \\
\hline Deltoid (n=20) & $12 / 20(60 \%)$ & $4.6 \pm 3.7(4.4)$ & $18.9 \pm 5.8(17)$ \\
\hline
\end{tabular}

EMG $=$ electromyography, MRC $=$ Medical Research Council, MUAP $=$ motor unit action potential, $\mathrm{TA}=$ tibialis anterior, $\mathrm{MG}=$ medial gastrocnemius, $\mathrm{PL}=$ peroneus longus, $\mathrm{VL}=$ vastus lateralis, $\mathrm{IP}=$ iliopsoas, $\mathrm{FDI}=$ first dorsal interosseous, $\mathrm{APB}$ $=$ abductor pollicis brevis. The MUAP amplitude of the Vastus lateralis muscle was higher than the MUAP amplitude of the tibialis anterior and medial gastrocnemius and iliopsoas muscles $(p=0.005, p=0.005, p=0.040)$. The MUAP duration of vastus lateralis muscle was longer than the MUAP duration of tibialis anterior and medial gastrocnemius muscles $(p=0.005$, $p<0.001)$. The MUAP amplitude of deltoid muscle was higher than the MUAP amplitude of biceps brachii muscle $(p=$ $0.015)$ and the MUAP amplitude of triceps brachii muscle was higher than the MUAP amplitude of FDI muscle $(p=0.040)$. The MUAP duration of deltoid muscle was longer than the MUAP duration of FDI and biceps brachii muscles $(p=0.001, p$ $<0.001)$. MUAP duration of triceps brachii muscle was longer than the MUAP duration of FDI muscle $(p=0.025)$. Friedman test and Wilcoxon signed-rank test were used to compare MUAP amplitudes and duration of muscles. Boferroni correction was used for post hoc analysis. $P$ values $<0.05$ were considered statistically significant.

absent CMAP of the peroneal or posterior tibial nerve. Fifty-seven lower and 32 upper extremities were evaluated with needle EMG. Needle EMG could not be applied to one lower limb of a patient due to edema. Needle EMG findings of 352 muscles were analyzed. There was no upper extremity meeting the criteria of Group 1. Thirty-eight lower extremities were included in Group 1, and the needle EMG findings in the muscles of these extremities are shown in Table 1. In lower extremities included in Group 1, MUAP was not obtained in $13(37 \%)$ of 35 tibialis anterior, $21(57 \%)$ of 37 medial gastrocnemius, $3(50 \%)$ of 6 peroneus longus, $16(50 \%)$ of 32 vastus lateralis and $11(34 \%)$ of 32 iliopsoas muscles. Table 2 shows the needle EMG findings in the muscles of the Group 2extremities. The muscles with a higher rate of needle EMG abnormalities in the upper and lower extremities included in Group 2 were deltoid (60\%) and vastus lateralis (94\%) muscles, respectively. The MUAP duration of the deltoid muscle was significantly longer than the MUAP duration of the first dorsal interosseous (FDI) and biceps brachii muscles ( $p=$ 0.001 and $p<0.001)$. The MUAP duration of the vastus lateralis muscle was longer than the MUAP duration of the tibialis anterior and medial gastrocnemius muscles $(p=0.005$ and $p<0.001)$. Table 3 shows the needle EMG findings obtained from the analysis of two muscles together. Needle EMG was performed on the trapezius muscle in 27 of 29 patients. The needle EMG of $4(15 \%)$ of 27 patients revealed neurogenic findings in the trapezius muscle. PSWs or FPs were seen in 22 muscles of 8 patients (Two patients had 


\section{Table 3. Needle EMG findings obtained by analyzing two muscles together in Group 2 extremities}

\begin{tabular}{lc}
\hline & $\begin{array}{c}\text { Number of muscles with neurogenic needle EMG } \\
\text { findings / total number of muscles examined in } \\
\text { needle EMG (\%) }\end{array}$ \\
\hline Deltoid + FDI, Deltoid + APB & $7 / 20(35 \%), 2 / 8(25 \%)$ \\
Triceps brachii + FDI, Triceps brachii + APB & $5 / 18(28 \%), 2 / 8(25 \%)$ \\
Biceps brachii + FDI, Biceps brachii + APB & $6 / 30(20 \%), 2 / 8(25 \%)$ \\
VL + MG, VL + PL & $8 / 18(44 \%), 2 / 6(33 \%)$ \\
IP + TA, IP + PL, IP + MG & $8 / 14(63 \%), 2 / 6(33 \%), 6 / 13(46 \%)$ \\
\hline
\end{tabular}

EMG $=$ electromyography, $\mathrm{MRC}=$ Medical Research Council, $\mathrm{TA}=$ tibialis anterior, $\mathrm{MG}=$ medial gastrocnemius, $\mathrm{PL}=$ peroneus longus, $\mathrm{VL}=$ vastus lateralis, $\mathrm{IP}=$ iliopsoas, $\mathrm{FDI}=$ first dorsal interosseous, $\mathrm{APB}=$ abductor pollicis brevis.

post-polio syndrome). The severity score of PSWs and FPs was at most grade two. PSWs or FPs were observed in 10 medial gastrocnemius muscles of 7 patients (Two patients had post-polio syndrome). Fasciculation potentials were present in two tibialis anterior muscles of two patients. The findings of these two patients were not compatible with post-polio syndrome.

\section{DISCUSSION}

Late neuromuscular deterioration may occur later in polio survivors. Although the reason for this is unknown, excessive exercise or immune mechanisms, or chronic poliovirus infection may cause late neuromuscular deterioration $[1,5,6]$. Moreover, the death of motor neurons is thought to be faster than normal aging $[7,8]$. Physical therapy strategies to protect motor neurons or axons may provide protection from neuromuscular deterioration. Excessive effort and immobility leading to weight gain can trigger neuromuscular deterioration $[1,5]$. Patients should receive sufficient but not excessive physical therapy. Physical therapy strategies can be developed by considering the affected regions. Needle EMG can help identify clinically or subclinically involved regions [9]. It is not practical to examine all the muscles of a polio survivor with needle EMG as it is painful and time-consuming. Our primary goal in this study was to identify the regions involved in polio survivors by applying needle EMG to as few muscles as possible.

MUAPs are difficult to obtain from the plegic limb muscles. For this reason, we classified the extremities according to MRC scores. Among the muscles of the extremities with a mean MRC score of 2 or less, MUAP was not obtained in mostly vastus lateralis, medial gastrocnemius, and peroneus longus muscles. Therefore, iliopsoas and tibialis anterior muscles can be used primarily in detecting neurogenic MUAP. If neurogenic MUAP is present in these two muscles, it can be stated that the lumbosacral region is affected. Otherwise, other muscles can be examined with needle EMG. The low number of MUAPs obtained in some muscles of severely affected extremities was one of the limitations of this study, because the amplitude and duration of the MUAPs were analyzed from these few MUAPs.

In the Group 2 lower extremities, the muscle with the highest rate of neurogenic MUAPs was vastus lateralis followed by the iliopsoas and tibialis anterior muscles. Therefore, it may be an option to start the needle EMG study with the tibialis anterior muscle

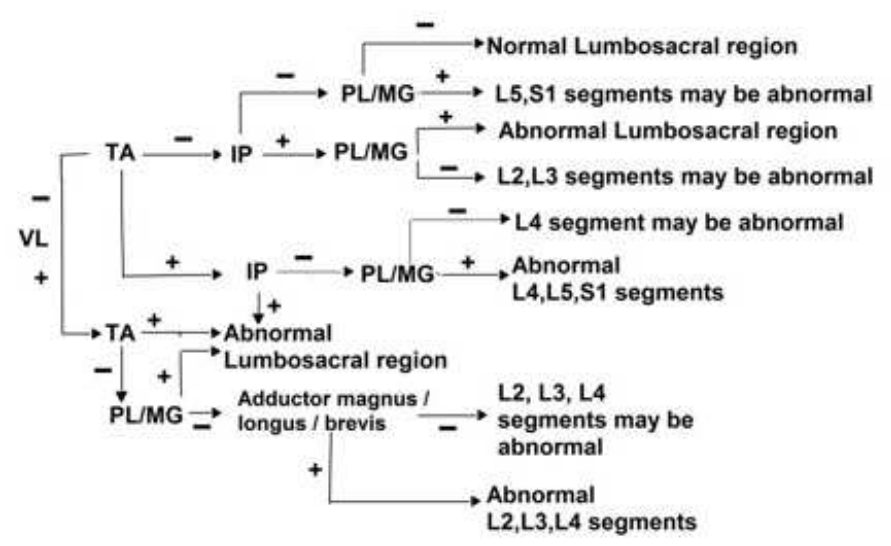

Fig. 1. Algorithm for muscle selection for needle EMG in the unaffected or mildly to moderately affected lower extremities of polio survivors. IP = iliopsoas, $\mathrm{MG}=$ medial gastrocnemius, $\mathrm{PL}=$ peroneus longus, $\mathrm{TA}=$ tibialis anterior, $\mathrm{VL}=$ vastus lateralis. 
and to continue the test with the iliopsoas muscle if a neurogenic abnormality is found in the tibialis anterior muscle. However, neurogenic needle EMG abnormalities were more common in the vastus lateralis muscle. In the case of neurogenic MUAP in two different muscles of an extremity innervated by different nerves and segments, we mentioned that this region was affected in the method section. Considering this principle, the algorithm for muscle selection for needle EMG in the clinically unaffected or mildly to moderately affected lower extremity is shown in Fig. 1. A similar algorithm for the muscles of the clinically unaffected or mildly to moderately affected upper extremity is shown in Fig. 2. Among the upper limb muscles, the highest rate of neurogenic MUAPs was present in the deltoid muscle followed by the triceps brachii and FDI muscles. Since the C6 segment innervates both the deltoid and triceps muscles, it is not practical to start needle EMG with deltoid and triceps brachii muscles. We think it would be appropriate to start needle EMG with deltoid and FDI muscles. Since this study was carried out in patients without peripheral nerve damage, it is useful to consider this situation when using these algorithms. According to histopathological and clinical studies, involvement patterns by regions and muscles have been discussed in the literature $[14,15]$. Similar to our study, in the literature, the involvement of the lower extremities was higher than the upper extremities [16]. It was reported that the upper lumbar spinal segments were affected more than the sacral segments [14]. Similarly, in this study, the muscles with the most neurogenic findings were the vastus lateralis, iliopsoas, and tibialis anterior muscles, respectively. In addition,

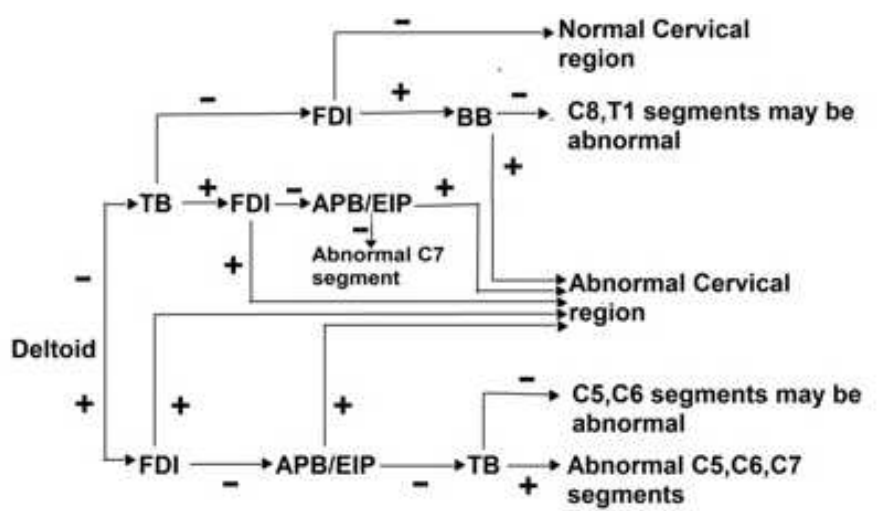

Fig. 2. Algorithm for muscle selection for needle EMG in the unaffected or mildly to moderately affected upper extremities of polio survivors. APB = abductor pollicis brevis, $B B=b i-$ ceps brachii, EIP = extensor indicis proprius, FDI = first dorsal interosseous, $\mathrm{TB}=$ triceps brachii. the presence of abnormal posterior tibial or peroneal nerve CMAP in $59 \%$ of patients and approximately $25 \%$ of the extremities where the nerve conduction study was performed may also support that the upper lumbar spinal segments were affected more than sacral segments. While Sharrard reported the most affected muscles in the upper extremity to be deltoid, triceps brachii, and pectoralis major muscles [14], another study stated that deltoid, triceps brachii, and biceps brachii muscles were mostly affected in the upper extremity [15]. Similarly, in our study, the most affected muscle in the upper extremity was the deltoid muscle. More neurogenic abnormalities in the deltoid muscle may indicate that the upper cervical segment is more affected than the lower cervical segment [14]. Contrary to this finding, the involvement of the biceps brachii muscle was less. This may be due to the shoulder muscles being prone to damage for carrying the hanging limb [15]. Another reason may be that the length of the spinal nuclear column for each muscle is different $[14,15]$. The muscle supplied by the short spinal nuclear column was more affected in poliomyelitis [14]. None of the patients had clinical involvement of cranial nerves. However, four patients had neurogenic needle EMG findings in the trapezius muscle. This finding was important since it showed that the bulbar region was affected slightly. Similar to the literature, in our study, $28 \%$ of patients and $6 \%$ of the muscles examined showed signs of active denervation [17]. Active denervation findings were mostly observed in the medial gastrocnemius muscle. We could not explain why this muscle was more involved, and this finding needs to be confirmed by further studies. This finding may be explained by the fact that the most common radiculopathies are L5 and S1 radiculopathies $[5,18]$.

\section{Limitations}

As we mentioned, the evaluation of a small number of MUAPs in some muscles of the severely affected extremities was one of the limitations of the study. The absence of needle EMG findings of muscles such as abductor digiti quinti, pronator teres, adductor magnus, or sternocleidomastoid was another limitation. In addition, the number of peroneus longus and abductor pollicis brevis muscles examined by needle EMG was low. We think that this algorithm can be improved with further studies in which more muscles 
are analyzed with needle EMG in polio survivors. Finally, since the trapezius muscle is innervated by both the 11th cranial nerve and the cervical segments, needle EMG abnormalities in the trapezius muscle may not indicate bulbar involvement. A study of poliomyelitis involving muscles that clearly indicate bulbar involvement, such as the tongue muscle, may be interesting.

\section{CONCLUSION}

We presented an algorithm for the muscle selection for needle EMG in polio survivors. Thus, clinically or subclinically affected regions and muscles can be determined practically.

\section{Authors' Contribution}

Study Conception: HF, İÖ; Study Design: HF; Supervision: HF, ZA; Funding: HF; Materials: HF, İÖ, ZA; Data Collection and/or Processing: HF, İÖ, ZA; Statistical Analysis and/or Data Interpretation: HF, IÖ, ZA; Literature Review: HF, İÖ, ZA; Manuscript Preparation: HF, İÖ, ZA and Critical Review: HF, İÖ, ZA.

\section{Conflict of interest}

The authors disclosed no conflict of interest during the preparation or publication of this manuscript.

\section{Financing}

The authors disclosed that they did not receive any grant during conduction or writing of this study.

\section{REFERENCES}

1. Agre JC, Rodriquez AA, Tafel JS. Late effects of polio: critical review of the literature on neuromuscular function. Arch Phys Med Rehabil 1991;72:923-31.
2. Horstmann DM. Epidemiology of poliomyelitis and allied diseases - 1963. Yale J Biol Med 1963;36:5-26.

3 . Bodian D. Histopathological basis of clinical findings in poliomyelitis. Am J Med 1949;6:563-78.

4. Kidd D, Williams AJ, Howard RS. Poliomyelitis. Postgrad Med J 1996;72:641-7.

5. Lo JK, Robinson LR. Postpolio syndrome and late effects of poliomyelitis. Part 1. Pathogenesis, biomechanical considerations, diagnosis, and investigations. Muscle Nerve 2018;58:7519.

6. Jubelt B, Cashman NR. Neurological manifestations of the post-polio syndrome. Crit Rev Neurobiol 1987;3:199-200.

7. McComas AJ, Quartly C, Griggs RC. Early and late losses of motor units after poliomyelitis. Brain 1997;120:1415-21.

8. Stalberg E, Grimby G. Dynamic electromyography and muscle biopsy changes in a 4-year follow-up: study of patients with a history of polio. Muscle Nerve 1981;4:524-8.

9. Fidancı H, Öztürk İ, Köylüoğlu AC, Buturak Ş, Arlıer Z. Pattern of muscle involvement according to needle electromyography findings in clinically unaffected extremities of polio survivors with lower extremity weaknesses. J Surg Med 2019;3:635-9.

10. Kleyweg R, Van der Meche F, Schimtz P. Interobserver agreement in the assessment of muscle strength and functional abilities in Guillain-Barre syndrome. Muscle Nerve 1991;14:1103-9.

11. Aids to the examination of peripheral nervous system. Memorandum No.45. London, HMSO. 1976.

12. Chen S, Andary M, Buschbacher R, Del Toro D, Smith B, So $\mathrm{Y}$, et al. Electrodiagnostic reference values for upper and lower limb nerve conduction studies in adult populations. Muscle Nerve 54: $371-7$.

13. Daube JR, Rubin DI. Needle electromyography. Muscle Nerve 2009;39:244-70.

14. Sharrard WJ. The distribution of the permanent paralysis in the lower limb in poliomyelitis; a clinical and pathological study. J Bone Joint Surg Br 1955;37:540-58.

15. Kumar K, Kapahtia NK. The pattern of muscle involvement in poliomyelitis of upper limb. Int Orthop 1986;10:11-5.

16. Ravits J, Hallett M, Baker M, Nilsson J, Dalakas MC. Clinical and electromyographic studies of postpoliomyelitis muscular atrophy. Muscle Nerve 1990;13:667-74.

17. Şenol MG, Kaplan C, Ozdağ F, Saraçoğlu M. How long denervation take in poliomyelitis? Or is it a lifetime? Neurosci Rural Pract 2018;8:511-5.

18. Tarulli AW, Raynor EM. Lumbosacral radiculopathy. Neurol Clin 2007;25:387-405. 